\title{
Criminologie
}

\section{Traitement des jeunes délinquants : modèle d'appariement selon le niveau conceptuel}

\section{Ronald Brill}

Volume 11, numéro 1, 1978

Centres d’accueil du Québec : accréditation et évaluation

URI : https://id.erudit.org/iderudit/017082ar

DOI : https://doi.org/10.7202/017082ar

Aller au sommaire du numéro

Éditeur(s)

Les Presses de l'Université de Montréal

ISSN

0316-0041 (imprimé)

1492-1367 (numérique)

Découvrir la revue

Citer cet article

Brill, R. (1978). Traitement des jeunes délinquants : modèle d'appariement selon le niveau conceptuel. Criminologie, 11(1), 46-64.

https://doi.org/10.7202/017082ar d'utilisation que vous pouvez consulter en ligne.

https://apropos.erudit.org/fr/usagers/politique-dutilisation/ 


\section{TRAITEMENT DES JEUNES DELINQUANTS : MODELLE D'APPARIEMENT SELON LE NIVEAU CONCEPTUEL *}

Ronald Brill

Ce qui apparaît nécessaire dans le domaine correctionnel, c'est un modèle théorique qui reconnaîtrait non seulement la diversité parmi les délinquants et parmi les types de traitement ; mais qui suggère aussi le moyen de coordonner ces différences entre clients avec les différents types d'interventions, ceci afin d'utiliser avec plus d'efficacité les ressources de traitement qui existent déjà dans ce domaine. Le modèle d'appariement du niveau conceptuel (Hunt, 1971) paraît offrir cette charpente. La théorie du niveau conceptuel, n'a été que récemment appliquée à ce domaine et n'avait pas été particulièrement développée pour la délinquance en tant que phénomène ou pour les délinquants en tant qu'individus. Elle a été plutôt utilisée en tant que théorie générale du développement de la personnalité ; celle-ci a été décrite en termes de quatre étapes progressives ou \&systèmes > d'organisation de la personnalité, chaque étape résultat de conditions ayant trait à la façon dont les parents élèvent leurs enfants (Harvey, Hunt, et Schroder, 1961). Chaque étape est aussi fonction des conditions de l'environnement qui faciliterait le fonctionnement optimal ou qui produirait de la tension, done un fonetionnement pathologique. De plus, cette théorie tient en considération les conditions nécessaires à la provocation, dans chaque système conceptuel, de la croissance vers plus de complexité et de maturité.

Hunt a continué à utiliser ces concepts, mais les a modifiés et développés en une théorie de base qui est soumise à des applications intensives dans le cadre scolaire avec des jeunes dont les âges varient de douze à dix-huit ans. Malgré que le développement conceptuel soit vu comme un processus continu, Hunt décrit trois stades de développement suivant l'âge des adolescents.

* Cet article s'inscrit dans le cadre d'une évaluation de Boys' Farm par le Groupe de recherche sur l'inadaptation juvénile de l'Université de Montréal et subventionné par: ministère des Affaires sociales du Québec, ministère du Solliciteur général du Canada et Foundation Boys' Farm.

Communication présentée à la première conférence de l'Association internationale đu traitement différentiel, Rensselaerville, N.Y., le 28 mars 1977. 
Le schéma I montre ces stades de développement, A, B, et $\mathbf{C}$, et leurs rapports aux dimensions du niveau conceptuel : concret abstrait, immaturité-maturité, simplicité-complexité conceptuelle.

SCHEMA I

Stades du développement conceptuel

\begin{tabular}{|c|c|c|c|}
\hline $\begin{array}{l}\text { Niveau } \\
\text { conceptuel }\end{array}$ & Etape & $\begin{array}{c}\text { Orientation } \\
\text { soi-autre }\end{array}$ & $\begin{array}{c}\text { Caractéristiques } \\
\text { d'étape }\end{array}$ \\
\hline Très bas & A & & $\begin{array}{l}\text { Centré en soi, phase } \\
\text { non-organisée avant l'in- } \\
\text { corporation de stan- } \\
\text { dards culturels }\end{array}$ \\
\hline Bas & B & & $\begin{array}{l}\text { Apprendre les règles de } \\
\text { base ou standards cul- } \\
\text { turels qui s'appliquent } \\
\text { a tous }\end{array}$ \\
\hline Elevê & C & & $\begin{array}{l}\text { Apprendre à se con- } \\
\text { naître et pourquoi l'on } \\
\text { est distinct des stan- } \\
\text { dards généralisés }\end{array}$ \\
\hline
\end{tabular}

(Abrégé de Hunt, 1971, p. 20)

Caractéristiques de chaque stade conceptuel

L'étape du développement la plus primitive et la plus concrète (étape A) est généralement caractérisée par le manque d'intériorisation des croyances culturelles et des valeurs normalement transmises par les parents ou autres personnes d'importance, ainsi que par un essai pour définir son moi par opposition aux autres. L'orientation résultant de ceci est une position concrète, antiautorité dans laquelle le négativisme apparaît par réaction aux suggestions des autres, ceci parce qu'elles sont interprétées comme subjugation. De plus, étant donné que ces personnes n'ont pas encore intériorisé de normes concernant le bien et le mal, ils voient les autres d'un œil égocentrique, ou comme des sources potentielles de gratification, sans vouloir rien donner en retour. Cette étape du développement n'est pas normalement vue comme un stade distinct, étant donné que les enfants progressent nor- 
malement à l'étape suivante. L'étape d'orientation A devient particulièrement évidente dans les circonstances où les conditions nécessaires à l'intériorisation des normes et valeurs ne sont pas fournies.

La deuxième étape du développement du niveau conceptuel (étape B) est vue comme étant le moment d'intériorisation des normes et valeurs de la société et des parents qui peuvent s'appliquer au sujet lui-même comme aux autres. Cette étape d'interprétation du monde implique des croyances non-critiques, noninterrogées. Les événements et les gens sont interprétés comme étant bons ou mauvais dépendant de la manière dont ils sont perçus. On pourrait dire que ce stade est une étape pro-autorité dans laquelle le sujet s'attend à ce que les adultes, qu'il voit comme étant bons ou justes, lui indiquent comment se comporter et définissent pour lui son monde par des prescriptions spécifiques.

L'étape suivante du développement interpersonnel (étape C) est atteinte lorsque le sujet se fie moins aux sources d'autorité, qu'il planifie son devenir comme un être indépendant et responsable et qu'il établit des perspectives et des lignes de conduite autonomes. Pendant cette étape la personne évalue à nouveau ses croyances et elle commence à voir le monde d'après ses propres conceptions plutôt qu'à partir de celles adoptées par les personnes en autorité. Pendant ce stade du développement les sentiments personnels jouent un rôle plus important dans les décisions ; auparavant, le comportement et les perceptions n'étaient que la résultante de critères extérieurs du bien et du mal.

\section{Modele d'appariement du niveau conceptuel}

Depuis sa première formulation, la théorie du niveau conceptuel a toujours tenté d'intégrer les composés personne $(\mathrm{P})$ et environnement $(E)$ dans la formule classique de Lewin : le comportement est une fonction de la personne et de son environnement : $C=F(P, E)$. Ce point de vue interactionniste a été utilisé dans la caractérisation des étapes du développement, et il est présentement utilisé pour formuler des prescriptions pour le fonctionnement optimal des sujets ayant atteint chaque étape, et pour amener des changements de stades conceptuels. Afin de venir en aide à celui qui formule le programme de traitement, une théorie du développement doit spécifier les besoins d'environnement des délinquants ayant atteint les différents stades de dé- 
veloppement et elle doit distinguer entre les besoins immédiats du client et ses besoins à long terme. Le modèle d'appariement du niveau conceptuel décrit les différences entre les personnes d'après leur complexité conceptuelle et leur orientation motivante. L'aspect de l'environnement qui s'apparente le plus directement à cette caractéristique des personnes est décrit par Hunt (1971) comme étant le degré de structure et par Schroeler, Driver et Streufert (1967) comme étant le degré de complexité. Le terme de degré de structure a été utilisé pour se rapporter au montant et à la diversité des composés informationnels, présents dans l'environnement, que l'individu peut utiliser en évaluant et/ou en formulant des réponses à cet environnement. Plus haute est la structure, plus ces éléments peuvent être considérés comme étant spécifiés de façon organisée et cohérente. Du point de vue de la perspective d'un programme de traitement, la structure se rapporterait au degré d'ordre, de clarté, d'organisation et de support que vivent les résidents d'une institution. L'aspect le plus important du degré de structure, pour l'individu qui est traité ou le groupe qui traite, c'est le montant de responsabilité qu'offre le programme du traitement. Mais ce qui apparaît être aussi important c'est le degré de spécificité et d'organisaton de l'interaction entre celui qui traite et le traité. Les environnements à haute structure sont concentrés autour de celui qui traite; ils impliquent la mise au point d'un contenu spécifique et ils spécifient des attentes particulières. Les environnements à basse structure seraient plus déterminés par les clients ; ils impliqueraient moins d'organisation dans les interactions mutuelles, et ils signifieraient la négociation des attentes mutuelles entre les employés et les clients.

A partir d'une connaissance du niveau conceptuel d'une personne, il est possible de préciser des prescriptions spécifiques concernant la nature de l'environnement; ceci afin de faciliter le fonctionnement optimal du sujet et d'encourager au maximum son développement (Hunt, 1971). Le schéma II illustre la relation entre le besoin de structure, tel que déterminé par la connaissance de son niveau conceptuel et le niveau approprié de structure que l'environnement doit pouvoir assurer.

A cause de la nature concrète et égocentrique des personnes à basse structure conceptuelle, celles-ci devraient profiter plus d'une approche à structure élevée. Les personnes à haute ca- 
SCHEMA II

Modele d'appariement

\begin{tabular}{|l|c|c|c|}
\hline $\begin{array}{l}\text { Besoin du } \\
\text { client }\end{array}$ & $\begin{array}{c}\text { A besoin de } \\
\text { beaucoup } \\
\text { de structure } \\
\text { conceptuel } \\
\text { Stade } \\
\text { A }\end{array}$ & $\begin{array}{c}\text { Stade } \\
\text { B }\end{array}$ & $\begin{array}{c}\text { A besoin de } \\
\text { peu } \\
\text { de structure } \\
\text { Stade } \\
\text { Cas }\end{array}$ \\
$\begin{array}{l}\text { Degré de } \\
\text { structure }\end{array}$ & $\begin{array}{c}\text { Normes très } \\
\text { claires, concrètes } \\
\text { support tangible }\end{array}$ & $\begin{array}{c}\text { Organisée, } \\
\text { qui supporte } \\
\text { a petits pas }\end{array}$ & $\begin{array}{c}\text { Autonomie élevée } \\
\text { pression normar } \\
\text { tive basse }\end{array}$ \\
\hline Elevé & & Bas \\
\hline
\end{tabular}

(Adapté de Hunt, 1974)

TABLEAU I

Résultats attendus des combinaisons d'étape et d'environnement

\begin{tabular}{|c|c|c|c|}
\hline \multirow{2}{*}{ Environnements } & \multicolumn{3}{|c|}{ Appariement étape - environnement } \\
\hline & Optimal & Sub-optimal & Super-optimal \\
\hline $\begin{array}{l}\text { Non clair } \\
\text { normative ou } \\
\text { inconsistant }\end{array}$ & & $\mathbf{A}$ & \\
\hline $\begin{array}{l}\text { Clairement organise } \\
\text { en dedans de la } \\
\text { structure normative }\end{array}$ & $\mathbf{A}$ & B & \\
\hline $\begin{array}{l}\text { Autonomie en dedans } \\
\text { de la } \\
\text { structure normative }\end{array}$ & B & $\mathrm{c}$ & $\mathbf{A}$ \\
\hline $\begin{array}{l}\text { Autonomie élevée / } \\
\text { basse pression } \\
\text { normative }\end{array}$ & C & & B \\
\hline $\begin{array}{l}\text { Accent sur la } \\
\text { mutualité }\end{array}$ & & & $\mathrm{C}$ \\
\hline Résultats attendus & $\begin{array}{l}\text { Progression } \\
\text { vers l'étape } \\
\text { suivante }\end{array}$ & $\begin{array}{c}\text { Rapprochement } \\
\text { de l'étape sui- } \\
\text { vante } A, B \text { ou } C\end{array}$ & $\begin{array}{l}\text { Plus de progres- } \\
\text { sion pour A et C, } \\
\text { rapprochement } \\
\text { transitionnel } \\
\text { pour l'étape B }\end{array}$ \\
\hline
\end{tabular}

(Adapté de Hunt, 1971, p. 22) 
pacité conceptuelle, par contre, sont complexes et plus en mesure de développer des nouveaux concepts et elles sont ainsi plus en mesure de s'adapter à des environnements différents ou changeants. Elles peuvent mieux profiter d'une structure flexible ou être moins affectées par les changements de structure.

\section{Appariement et développement conceptuel}

En référence aux différents stades conceptuels, il est possible de définir le degré de structure qui sera optimal, suboptimal, ou super-optimal en termes de travail conceptuel qui doit être accompli pour assurer la progression vers un niveau supérieur. Le tableau I décrit ces relations et prescriptions.

D'après la nature conceptuelle simple et très concrète d'une personne d'étape $A$, un environnement apparié est vu comme ayant une structure élevée, c'est-à-dire claire, consistante, bien organisée. Ces conditions provoquent la progression vers l'étape $B$ en rendant possible à l'individu de l'étape $\mathbf{A}$ la compréhension des normes et de la situation et leurs acceptations comme lignes de conduite pour son propre comportement. Un environnement, dont les normes ne sont pas consistantes ou ne sont pas présentées de façon claire et concrète, empêche le changement d'étape, car l'individu de l'étape $\mathrm{A}$ est incapable de comprendre et de résoudre seul les incohérences. Un blocage à l'étape A provient d'un environnement qui exige de l'autonomie des jeunes : c'est-à-dire de l'indépendance et une certaine capacité pour produire des standards personnels.

Pour l'individu de l'étape $B$, qui opère déjà à l'aide d'une certaine échelle de valeurs personnelles, le changement vers l'étape $\mathrm{C}$ requiert qu'il produise des standards personnels et qu'il prenne des décisions reposant sur ces standards. Donc, l'environnement optimal doit encourager le détachement des standards reposant uniquement sur les besoins personnels, mais sans dissociation nette des normes précédemment acceptées. L'environnement qui renforce la dépendance envers les normes acceptées maintient par le fait même la structure conceptuelle du stade B et il ne provoque plus la croissance. L'environnement qui ne contient aucune structure, mais qui suppose des standards personnels, arrête la croissance pour l'individu de l'étape B, étant donné qu'il est encore incapable de fonctionner sans ces normes et il essaie alors de trouver de nouveaux moyens pour faire face 
à la situation. La structure conceptuelle qui en résulte est une articulation incomplète des étapes $\mathrm{B}$ et $\mathrm{C}$, en ce sens qu'elle retient certaines facettes de chacune de ces étapes.

L'environnement optimal pour l'individu du stade $\mathrm{C}$ devrait encourager le développement d'une capacité de distinction par rapport aux normes externes et il devrait désaccentuer les pressions normalisantes et augmenter les occasions d'indépendance. Un environnement qui renforce la soumission aux normes empêche la croissance, car il détourne l'attention du développement de standards personnels de la participation et de la compréhension des sentiments et des valeurs des autres.

\section{Application du modele d'appariement du niveau conceptuel}

a) Recherche en éducation

Les expériences, jusqu'à présent, ont tenté d'identifier les personnes de niveau conceptuel élevé et inférieur et de les placer dans des conditjons d'étude variant suivant le degré de structure, tout en tenant compte de l'intelligence. McLachlan et Hunt (1973) ont démontré l'efficacité différentielle d'un apprentissage par découverte avec des étudiants de bas niveau conceptuel; ceux-ci apprennent décidément mieux dans le cadre d'un cours structuré, tandis que les étudiants ayant un niveau conceptuel élevé apprennent mieux par une méthode par découverte guidée. Les étudiants d'un niveau conceptuel élevé apprenaient aussi bien sous les deux types de conditions structurelles. La présentation d'une règle avant ou après l'exposition d'exemples a aussi démontré la production d'effets différentiels avec des groupes différents de niveau conceptuel (Tomlinson et Hunt, 1971). Les étudiants de haut niveau conceptuel fonctionnaient bien sous toutes les conditions mais leur performance était moins bonne dans des conditions de haute structure. Une étude récente sur les effets des différentes approches pour aider les étudiants à comprendre les gens d'un autre pays (Salyachivin, 1972) a démontré que les étudiants de bas niveau conceptuel sont plus suggestibles si l'approche est structurée.

La pertinence du modèle d'appariement du niveau conceptuel a aussi été démontrée par des études où psychothérapeutes et patients alcooliques étaient assortis. McLachlan (1972) a démontré que plus élevé était le niveau conceptuel du thérapeute de groupe, plus son style de thérapie était non-directif. Lorsque 
les clients à bas niveau conceptuel étaient appariés avec les thérapeutes à bas niveau conceptuel et les patients à haut niveau conceptuel appariés aux thérapeutes à niveau conceptuel élevé, les patients se jugeaient comme étant plus améliorés que les patients non appariés, et montraient aussi plus d'amélioration dans leur comportement envers l'acool un an plus tard (McLachlan, 1974). Après révision des recherches en psychothérapie, Posthuma et Carr (1975) ont conclu que l'appariement de niveaux conceptuels entre patients et thérapeutes améliorait beaucoup la probabilité de succès de la thérapie et les résultats proprement dits. Ils suggèrent, de plus, que l'appariement de niveau conceptuel des membres d'un groupe apparaissait améliorer le processus et le résultat de thérapie.

On a aussi trouvé d'autres informations pour le modèle d'appariement du niveau conceptuel lors de l'évaluation des programmes «upward bound ». Dans ces programmes, il s'agissait de motiver des étudiants du secondaire, provenant de quartiers pauvres, à continuer leurs études au niveau collégial. Pour encourager le changement d'attitude, des étudiants ont été dirigés vers des programmes résidentiels d'été organisés par des collèges, partout aux États-Unis. Ces programmes ont été évalués suivant le questionnaire de climat de programme qui s'intéressait à la chaleur et à la structure des programmes (Hunt, Hardt et Victor, 1968).

Les programmes ont été caractérisés comme étant hauts ou bas en termes de structure, et les groupes d'étudiants de chacun comme ayant un niveau conceptuel élevé ou inférieur. Des groupes d'étudiants, qui étaient appariés à la structure de leurs programmes, ont démontré qu'ils avaient beaucoup plus changé leur motivation à continuer leurs études que ceux qui ne l'étaient pas (Hunt et Hardt, 1967). Ils avaient aussi changé dans l'évaluation de leur capacité à graduer du collège, ainsi que dans leur motivation à comprendre les autres.

\section{b) Une recherche dans le domaine du traitement des jeunes délinquants}

La plus récente application du modèle d'appariement $d u$ niveau conceptuel a eu lieu dans le domaine du traitement des délinquants institutionnalisés (Brill, 1977). Vu que cette étude 
se rapporte directement au présent sujet, nous la décrivons en détail.

Quarante-trois garçons délinquants ont été placés dans deux programmes de traitement résidentiels d'après leur niveau conceptuel. La structure des programmes a été choisie de telle façon que quatre sous-groupes définis théoriquement soient constitués. Deux sous-groupes, ont été appariés théoriquement suivant le degré de structure des programmes : les garçons du stade $\mathbf{A}$ ont été placés dans un programme à haute structure, et les garçons les plus mûrs, ceux de l'étape $B$, dans le programme le moins structuré. Quelques garçons placés dans chaque programme ont été aussi théoriquement non-appariés, des garçons de l'étape A ont été placés dans le programme à basse structure et des garçons de l'étape $B$ dans le programme à haute structure. Le progrès de ces quatre groupes a été subséquemment évalué au moment de leur retour dans la communauté. Ces échantillons étant petits, la performance des deux groupes appariés est comparée à celle des deux groupes non-appariés. Le tableau II illustre ce modèle de recherche.

Une hypothèse a été formulée à l'effet que les groupes appariés profitent plus du programme que les groupes non-appariés. Par ailleurs, la plus grande amélioration se rencontre chez les garçons de l'étape $A$ appariés et non-appariés que chez les garçons de l'étape $B$ appariés ou non. Des mesures des résultats ont été

TABLEAU II

Comparaisons des groupes de l'étude

\begin{tabular}{|c|c|}
\hline Stades conceptuels & $\begin{array}{l}\text { Comparaisons des groupes } \\
\text { pendant le traitement residentiel }\end{array}$ \\
\hline \multirow{3}{*}{$\begin{array}{l}\text { A } \\
\text { B }\end{array}$} & Structure du programme \\
\hline & $\begin{array}{c}\text { Structure élevée } \\
\text { Appariés } \longleftarrow \text { vs } \longrightarrow \text { Basse structure } \\
\text { Non-appariés } \longleftarrow \text { vs } \longrightarrow \text { appariéés }\end{array}$ \\
\hline & $\begin{array}{l}\text { Comparaisons durant la période de } \\
\text { suite post-résidentielle }\end{array}$ \\
\hline $\begin{array}{c}\text { A \& B } \\
\text { (combinés) }\end{array}$ & $\begin{array}{l}\text { Appariés } \longleftarrow \text { vs } \longrightarrow \\
\text { en résidence }\end{array} \begin{array}{l}\text { non-appariés } \\
\text { en résidence }\end{array}$ \\
\hline
\end{tabular}


prises dans les dossiers sur le comportement pendant la période du traitement résidentiel et durant la période de surveillance dans la communauté. Ces mesures consistaient en divers événements : (placement en détention, fugues, journées passées en dehors du programme conséquemment à ces incidents, et une évaluation générale du succès ou de l'échec du traitement : l'échec comporte le transfert à un autre programme résidentiel, le retour en résidence à la suite d'un placement dans la communauté, la fugue sans retour et le déferré au système de justice criminelle pour adultes). Un ensemble de mesures écrites sur l'ajustement psychologique, les attitudes et le comportement ont été recueillis avant le traitement résidentiel ainsi qu'à la fin, afin d'évaluer les changements produits par le traitement.

Pendant les vingt-quatre mois qu'a duré le traitement résidentiel, les programmes ont été évalués quatre fois par des observateurs à partir de deux procédés relatifs au climat social. Parallèlement les évaluations sur six échelles, se rapportant particulièrement à la structure des programmes, ont été comparées. Une procédure additionnelle a été utilisée pour comparer la structure des programmes : elle consiste à étudier les cahiers quotidiens de présence des pavillons, ceci pendant cinq périodes d'un mois, grâce à un évaluateur neutre et à quatre dimensions se référant à la structure relative des programmes. Les deux programmes se sont avérés différents sur trois des six échelles se rapportant à l'environnement ainsi que sur trois des quatre dimensions des cahiers de présence des pavillons. Donc les deux pavillons démontraient des niveaux différents d'organisation, de consistance, de planification et de support pour les garçons. Les tableaux III et IV donnent le nombre moyen des incidents comportementaux et le nombre de jours passés hors-programme pour tous les sous-groupes pendant le séjour institutionnel.

Les résultats présentés aux tableaux III et IV prouvent que les garçons de l'étape A non-appariés avaient deux fois plus d'incidents comportementaux que les garçons de l'étape $A$ appariés, et qu'ils passaient trois fois plus de temps, en moyenne, hors-programme à cause de ces incidents. En effet, le temps moyen de résidence pour les groupes de l'étape A non-appariés et appariés étaient de 11,8 et 12,7 mois respectivement ; le temps moyen hors-programme comptait respectivement pour $27,3 \%$ et $7,6 \%$ du temps total passé en résidence. Aucune différence n'est 
apparente entre les groupes de l'étape B appariés et non-appariés. Ces résultats supportent l'hypothèse, c'est-à-dire que l'appariement est plus crucial pour les individus de l'étape A que pour ceux du stade $B$.

Dans un effort pour estimer l'impact de l'appariement au besoin de structure sur l'ajustement post-traitement des échantillons des sous-groupes originaux ont été suivis pendant une période moyenne de huit mois. Les échantillons appariés étaient combinés pour les comparer aux échantillons non-appariés. Les échantillons appariés avaient donc une plus grande proportion de garçons sans incidents comportementaux et une plus grande proportion de ces garçons ont eu plus de succès que ceux des

\section{TABLEAU III}

Nombre moyen d'incidents comportementaux par sous-groupes du programme et niveau conceptuel

\begin{tabular}{|l|c|c|c|c|}
\hline & Moyenne & Ecart type & $\mathrm{U}^{2}$ & $\mathrm{p}$ \\
\hline Stade A & & & & \\
Appariés & 2,07 & 3,12 & 33,5 & $<, 05$ \\
Non-appariés & 4,56 & 4,07 & & \\
Stade B & & & & \\
Appariés & 2,36 & 2,91 & 46,5 & n.s. \\
Non-appariés & 2,44 & 3,05 & & \\
\hline
\end{tabular}

1. Test U de Mann Withney.

TABLEAU IV

Jours en dehors du programme par sous-groupes de programme et niveau conceptuel

\begin{tabular}{|l|c|c|c|c|c|c|}
\hline & Etendue & Médiane & Moyenne & $\begin{array}{c}\text { Ecart } \\
\text { type }\end{array}$ & $U^{3}$ & $p$ \\
\hline Stade $A$ & & & & & & \\
Appariés & $0-230$ & 3 & 28,93 & 62,70 & 15,5 & $<, 01$ \\
Non-appariés & $0-349$ & 67 & 97,44 & 121,82 & & \\
Stade B & & & & & & \\
Appariés & $0-200$ & 4 & 27,0 & 58,76 & 46 & n.s. \\
Non-appariés & $0-94$ & 3 & 30,44 & 39,68 & 46 \\
\hline
\end{tabular}

1. Test U de Mann Withney. 
échantillons non-appariés. Les tableaux $\mathrm{V}$ et $\mathrm{VI}$ montrent ces différences de proportion.

Il est peut-être surprenant que les effets initiaux observés se sont maintenus malgré que plusieurs des garçons étaient probablement non-appariés pendant la période de résidence postrésidentielle. Ces effets suggèrent fortement que l'appariement initial a beaucoup contribué à changer l'attitude et le mode de vie de ces garçons. Ces effets sont semblables à ceux rapportés par McLachlan (1974) dans son évaluation des patients alcooliques qui ont participé à une thérapie de groupe menée par des thérapeutes dont le niveau conceptuel était apparié ou nonapparié. Pour les patients appariés à leur thérapie de groupe, $70 \%$ ont guéri alors que seulement $50 \%$ de ceux qui étaient non-appariés ont guéri. Quand le niveau conceptuel des patients a été apparié au thérapeute et à l'environnement après soins, $77 \%$ ont guéri ; quand le niveau conceptuel des patients était non-apparié à ces deux conditions, seulement $38 \%$ ont guéri.

\section{TABLEAU $\mathrm{V}$}

Fréquence d'incidents comportementaux pour les échantillons appariés et non-appariés *

\begin{tabular}{|l|c|c|}
\hline & \multicolumn{2}{|c|}{ Incidents de comportementaux } \\
\hline GROUPE & $\frac{0}{12}$ & $\frac{1}{1}$ \\
Appariés & 12 & 7 \\
Non-appariés & 7 & \\
\hline
\end{tabular}

* Probabilité de différences en proportions $p<, 025$, test de probabilité de Fisher.

TABLEAU VI

Evaluation de succès du traitement dans les échantillons appariés et non-appariés*

\begin{tabular}{|l|c|c|}
\hline & \multicolumn{2}{|c|}{ Evaluation du traitement } \\
\hline GROUPE & Succès & Echec \\
Appariés & 13 & 1 \\
Non-appariés & 7 & 8 \\
\hline
\end{tabular}

* Probabilité de différence en proportion $p<, 01$, test exact de Fisher. 
Une analyse de covariance à deux facettes des questionnaires administrés au moment de l'admission, et au moment du départ de résidence, n'a révélé aucun impact différentiel auprès des deux sous-groupes d'appariement du programme et du niveau conceptuel. Plusieurs effets importants indiquent que les garçons de l'étape $B$ changent plus vite et dans une direction plus positive que les garçons de l'étape $\mathrm{A}$. Ces résultats n'ont pas été inclus ici à cause du manque d'espace mais ils peuvent être lus dans Brill (1977). La comparaison entre sous-groupes sur plusieurs mesures de changement n'ont révélé aucune différence dans l'ensemble, mais les garçons de l'étape $\mathrm{B}$ montrent plus d'amélioration sur les échelles d'attitude, alors que les garçons de l'étape A montrent plus d'amélioration sur les échelles de comportement. Le tableau VII illustre ces changements entre les différents sousgroupes et niveaux conceptuels. Le tableau détaillé peut être consulté dans Brill (1977).

Ces résultats, c'est-à-dire les changements d'attitude pour les garçons de l'étape $B$ et les changements de comportements pour les garçons de l'étape $A$, ne sont pas surprenants. En effet, la théorie du niveau conceptuel suggère clairement que les individus du stade $B$ sont plus ouvertement dépendants des règlements et des personnes en autorité et qu'ils devraient donc avoir plus de préparation pour s'impliquer avec les éducateurs et s'attaquer à leurs problèmes personnels ainsi qu'à leurs problèmes plus généraux avec leur famille, l'école ou leurs pairs. La théorie suggère aussi qu'étant donné qu'ils sont plus complexes conceptuellement, que les adolescents du stade A, ils devraient pouvoir résoudre plus efficacement leurs problèmes, au niveau verbal ou conceptuel en discutant avec les éducateurs que les individus du stade $\mathrm{A}$.

Les différences entre les garçons des stades conceptuels supportent ce raisonnement puisque les garçons de l'étape A étaient évalués supérieurs sur les échelles indiquant leur méfiance face à l'autorité, leurs sentiments d'être incompris, malheureux et inquiets, mais ils désiraient être membres d'une bande des durs et ils recherchaient le sensationnel. Les différences entre les garçons des stades A et B, sur l'inventaire du comportement en classe indiquent que les garçons de l'étape $A$ montrent moins de confiance en eux-mêmes que les garçons de l'étape $B$, mais plutôt que d'être repliés sur eux-mêmes et non-impliqués, ils 
sont bruyants et ils cherchent à attirer l'attention (Brill, 1972). Hunt et Hardt (1965) présentent des résultats qui démontrent que les délinquants de l'étape $A$ trouvent que parler de leurs plans d'avenir est très troublant et qu'ils préfèrent être quelqu'un d'autre qu'eux-mêmes. Ces tendances démontrent que les garçons de l'étape A essaient de faire face à leurs difficultés en les évitant ou les niant et en exhibant un comportement anti-social et agressif. Il est donc facile de comprendre pourquoi ils ne réus-

TABLEAU VII

Changements significatifs dans les sous-groupes d'appariement du programme - niveau conceptuel indiqué par le test-t sur les mesures de changement choisies *

\begin{tabular}{|c|c|c|c|c|}
\hline & $\begin{array}{l}\text { Appariés } \\
\text { stade A }\end{array}$ & $\begin{array}{l}\text { Non- } \\
\text { appariés } \\
\text { stade A }\end{array}$ & $\begin{array}{l}\text { Appariés } \\
\text { stade B }\end{array}$ & $\begin{array}{l}\text { Non- } \\
\text { appariés } \\
\text { stade B }\end{array}$ \\
\hline $\begin{array}{l}\text { Variable } \\
\text { Justesse de soi } \\
\text { Estime de soi } \\
\text { Inventaire de Jesness } \\
\text { Mésadaptation sociale } \\
\text { Orientation en valeurs } \\
\text { Immaturité } \\
\text { Autisme } \\
\text { Aliénation } \\
\text { Agression manifeste } \\
\text { Retraitisme } \\
\text { Anxiété sociale } \\
\text { Répression } \\
\text { Dénégation } \\
\text { Index d'asocialité } \\
\\
\text { Inventaire du comportement } \\
\text { de Jesness (observateur) } \\
\text { Non-importunité } \\
\text { Amitié } \\
\text { Responsabilité } \\
\text { Considération } \\
\text { Indépendance } \\
\text { Rapport } \\
\text { Enthousiasme } \\
\text { Sociabilité } \\
\text { Conformité } \\
\text { Calme } \\
\text { Communicátion } \\
\text { Introspection } \\
\text { Contróle social } \\
\text { Controle de la colère }\end{array}$ & $\begin{array}{l}, 001 \\
, 05 \\
, 001 \\
, 01 \\
, 01 \\
, 001\end{array}$ & $\begin{array}{l}, 01 \\
, 01 \\
, 01 \\
, 05 \\
, 05 \\
, 001 \\
, 05 \\
, 01\end{array}$ & $\begin{array}{l}, 05 \\
, 01 \\
, 05 \\
, 01 \\
, 01 \\
, 01\end{array}$ & $\begin{array}{l}.001 \\
, 01 \\
, 01 \\
, 05\end{array}$ \\
\hline
\end{tabular}

* Les numéros dans le tableau indiquent la probabilité de changements de niveaux. 
sissent pas à s'améliorer puisqu'ils ne peuvent faire de retour sur eux-mêmes.

Il est donc apparent que le facteur crucial qui contribue à un comportement plus satisfaisant, chez les garçons du stade A appariés durant le traitement résidentiel, est l'effet stabilisateur du programme à haute structure; ceci afin que leurs attitudes sociales inadaptées ne soient pas exprimées en termes d'incidents comportementaux. Dans l'ensemble, la haute structure et le support reçus par ces garçons ont compensé leur incacapité à structurer leur propre expérience, ce qui en retour résulte en moins de frustrations et leur donne le pouvoir de développer plus de moyens pour faire face à leurs problèmes. D'un autre côté, les garçons de l'étape A non-appariés manquent de structure et de support pour organiser leur vie avec succès et ils subissent inévitablement plus de frustrations, ce qui conduit à un plus grand nombre d'incidents comportementaux et par la suite une attitude, déjà fortement mal ajustée envers eux-mêmes et envers l'autorité, se renforce. En ce qui concerne les garçons de l'étape B, ces variations dans le support ne sont pas aussi cruciales pour le succès de l'organisation de leur vie, cette situation s'explique par le fait qu'ils possèdent les ressources pour le faire dans n'importe quelle situation, et ceci assez bien pour prévenir les incidents comportementaux.

\section{Implications pour la recherche et la pratique}

Cette étude soulève de nombreuses questions pour les recherches futures, mais les résultats ont des implications assez claires pour permettre leur application. Plus important, cette étude démontre la valeur du placement des garçons de stade $\mathbf{A}$ dans un environnement à haute structure afin de récolter un ajustement immédiat appréciable ainsi que plus de succès dans la communauté. Les garçons non-appariés du stade $B$ ne montrent pas des résultats très négatifs dans un milieu à haute structure, tel que prédit, mais ils ont tout de même contribué à un nombre d'incidents comportementaux (tableau $V$ ) et d'échecs d'adaptation (tableau VI), deux fois plus important. Il semble que la prédiction d'une baisse de performance, pour les individus placés dans des conditions de haute structure, s'appliquerait plus particulièrement aux individus du stade $C$ et en transition entre les stades $B$ et $C$. Ces résultats s'expliquent par le fait que l'in- 
dividu du stade $\mathrm{C}$ a été décrit comme ayant non seulement plus de capacités, mais aussi plus d'aptitudes pour agir avec plus de responsabilités et d'autonomie que les individus du stade B. Malgré que les personnes du stade B ont été caractérisées comme étant dépendantes et comptant sur les règlements, elles sont théoriquement plus complexes et plus capables d'agir avec autonomie que les individus du stade $A$. Donc, on pouvait s'attendre à ce que dans des conditions très structurées et appropriées pour les garçons du stade $\mathrm{A}$, les garçons de l'étape B auraient réagi plus négativement qu'ils ne l'ont fait. Il semble, malgré que les garçons du stade B n'ont pas besoin d'une structure très forte pour fonctionner avec responsabilité, que celle-ci ne leur est pas aussi nuisible qu'elle le serait pour un garçon du stade $\mathrm{C}$. Malgré l'absence de recherches sur les effets néfastes d'un placement de jeunes délinquants à haut niveau conceptuel dans un environnement à structure élevée, cette prescription semble pertinente mais à investiguer systématiquement. Par ailleurs, plusieurs des études mentionnées plus haut, montrent que des individus à haut niveau conceptuel sont plus capables d'analyser les informations que les individus de niveau inférieur, et il y a beaucoup d'évidence à l'effet que les délinquants à haut niveau conceptuel sont plus capables d'agir avec plus de responsabilité et d'indépendance que ceux d'un niveau inférieur. Une étude exploratoire (Brill, 1973) impliquant l'évaluation de tous les garçons par le personnel, a indiqué une relation significative entre l'indépendance et le sens des responsabilités et un niveau conceptuel supérieur au stade B.

Les besoins de structure des garçons de différents stades conceptuels suggèrent non seulement que le traitement serait plus effectif s'il apparaissait dans un cadre où la structure du programme est appariée à ces besoins, mais aussi qu'il serait bénéfique d'apparier l'éducateur responsable du garçon suivant son degré de directivité. L'étude de McLachlan sur des thérapeutes directifs et non-directifs, qui étaient appariés ou non-appariés au niveau conceptuel de patients alcooliques, a démontré l'efficacité de cette approche.

Une autre implication du modèle d'appariement du niveau conceptuel pour la pratique est que l'appariement des besoins des clients avec la structure du programme devrait être continu d'un endroit de traitement à l'autre. Des cas peuvent être fréquemment 
transférés d'un milieu résidentiel à la communauté, pour y revenir par la suite, si le besoin s'en fait sentir; alors on s'attend à ce que le jeune puisse fonctionner dans un environnement auquel il est non-apparié et il s'ensuit donc un retour aux anciennes façons de faire face à ces frustrations. Il est essentiel de se rappeler que, dans la communauté, des jeunes ont plus de situations auxquelles ils doivent faire face, la famille, le foyer de groupe, l'école, le travail, le style de traitement du conseiller, etc., et on ne sait pas encore très bien quelle combinaison doit être appariée pour pouvoir continuer un traitement efficace dans la communauté. Du point de vue de la théorie du niveau conceptuel on s'attend à ce que les jeunes de l'étape $A$ puissent tolérer le moins le non-appariement, alors que les jeunes de l'étape $\mathbf{C}$ peuvent en tolérer plus. Si l'appariement peut être accompli de façon adéquate, le recyclage des cas peut être réduit et l'agence de traitement peut servir plus de clients avec plus d'efficacité.

Une dernière implication du modèle d'appariement du niveau conceptuel concerne la définition du type de traitement et le rôle de l'environnement dans le traitement. Le traitement est fréquemment conceptualisé comme pouvant être fait sur les clients, car le problème réside dans les clients eux-mêmes, plutôt que d'être le produit de l'interaction entre la nature du client et la nature de son environnement. Les clients diffèrent clairement, et leurs difficultés dès l'arrivée en institution, peuvent en relation directe avec le degré de non-appariement qu'ils ont reçu ailleurs - mais presque immédiatement l'interaction entre le besoin de structure du client et la structure du nouvel environnement peut agir pour confondre les problèmes originaux avec les nouveaux problèmes résultant du non-appariement dans l'environnement actuel. Si le personnel affecté au traitement perçoit le comportement du client comme venant du client lui-même, on ne se pose jamais la question à savoir si l'environnement est approprié. Dans de telles circonstances, les clients peuvent être classifiés comme étant non-traitables, malades ou psychopathes car ils n'agissent pas selon les espérances du personnel conduisant le programme.

\section{Conclusion}

Cet article débutait avec la suggestion que le domaine correctionnel a grandement besoin d'un cadre théorique pour coordonner les différences parmi les délinquants avec la variété 
d'approches thérapeutiques afin d'agrandir les bénéfices pour les délinquants et pour la société. Le modèle d'appariement du niveau conceptuel semble offrir une théorie de base cohérente pour le placement différentiel des jeunes dans des environnements communautaires ou résidentiels et pour le placement différentiel selon la structure des programmes existants et/ou les styles des thérapeutes. Alternativement, dès que les besoins de structure de la clientèle d'une agence de traitement sont connus, de nouveaux programmes, de nouvelles stratégies pourraient être conçus afin de rencontrer les besoins de ceux qui sont non-appariés aux programmes présents. Étant donné que les caractéristiques des clients, ainsi que des environnements, sont habituellement conceptualisés à un haut niveau d'abstraction, le modèle apparaitrait donc applicable pour coordonner les caractéristiques de l'individu ou des groupes de jeunes avec une grande variété de ressources de traitement, qu'elles soient conçues en termes de modèles thérapeutiques spécifiques comme l'analyse transactionnelle, la thérapie de réalité, etc., ou plus généralement, en termes de la structure prévue par le programme et/ou les dispensateurs du traitement.

\section{BIBLIOGRAPHIE}

BRILL, R., Comparaison of stage $A \& B$ delinquent youth on the Classroom Behavior Inventory, Unpublished data, 1972 (Available from author, Boys' Farm and Training School, Shawbridge, P.Q.).

BRILL, R., Comparaison of $C L$ stages $B$ and $B-C$ delinquent boys on staff ratings of independence and responsibility, Unpublished data, 1973 (Available from author, Boys' Farm and Training School, Shawbridge, P.Q.).

BRILL, R., Effects of residential program structure and conceptual level in treatment of delinquent boys, Unpublished doctoral dissertation, University of Toronto, 1977.

HARVEY, O.J., HUNT, D.E., and SCHROEDER, H.M., Conceptual Systems and Personality Organization, New York. John Wiley \& Sons, 1961.

HUNT, D.E., and HARDT, R.H., The role of conceptual level and program structure in summer $U_{p}$ ward-Bound programs, Paper presented at Eastern Psychological Association Meeting, Boston, April, 1967.

HUNT, D.E., HARDT, R.H., and VICTOR, J.B., Characterization of Upward-Bound: Summer and Academic Year, 1967-1968, Syracuse University Youth Development Center, 1968.

HUNT, D.E., Matching models in education, Toronto, Ontario Institute for Studies in Education, 1971. 
HUNT, D.E., and HARDT, R.H., Developmental stage, delinquency, and differential treatment », Journal of Research in Crime and Delinquency, $1965,2,20-31$.

MCLACHLAN, J.F.C., \& Benefit from group therapy as a function of patient-therapist match on conceptual level $\gg$, Psycho-therapy: Theory, Research and Practice, 1972, 9, 317-323.

McLACHLAN, J.F.C., Therapy strategies, personality orientation and recovery from alcoholism », Canadian Psychiatric Association Journal, 1974, 19, 25-30.

McLACHLAN, J.F.C., and HUNT, D.E., «Differential effects of discovery learning as a function of student conceptual level $\gg$, Canadian journal of Behavioral Science, 1973, 5, 152-160.

POSTHUMA, A.B. and CARR, J.E., \& Differentiation matching in psychotherapy 》, Canadian Psychological Review, 1975, 16, 35-43.

SALYACHIVIN, S., Change in international understanding as a function of similarity, conceptual level, and primacy, Unpublished doctoral dissertation, University of Toronto, 1972.

SCHRODER, H.M., DRIVER, M.J. and STREUFERT, S., Human information processing, New York, Holt, Rinehart and Winston, 1967.

TOMLINSON, P.D., and HUNT, D.E., «Differential effects of ruleexample order as a function of learner conceptual level $\gg$, Canadian Journal of Behavioral Science, 1971, 3, 235-237. 